01,11

\title{
Псевдоспинодаль при моделировании распада сплава методом Монте-Карло
}

\author{
(С) И.К. Разумов \\ Институт фризики металлов УрО РАН, \\ Екатеринбург, Россия \\ E-mail: rik@imp.uran.ru
}

(Поступила в Редакцию 11 мая 2016 г.)

На основании результатов моделирования методом Монте-Карло распада в простой системе с короткодействующим потенциалом притяжения примесных атомов выдвигается гипотеза о существовании псевдоспинодали, разделяющей области гомогенного и гетерогенного зародышеобразования на фазовой диаграмме.

Работа выполнена в рамках государственного задания ФАНО России (тема „Магнит“, № 01201463328).

DOI: 10.21883/FTT.2017.04.44261.181

\section{1. Введение}

В теории фазовых превращений в славах [1,2] различные типы кинетики распада классифицируют по положению параметров сплава (температура, состав) на фазовой диаграмме. Предел растворимости (бинодаль) определяется условием равенства химических потенциалов фаз, а предел устойчивости однородного состояния (спинодаль) определяется как граница выпуклого участка концентрационной зависимости свободной энергии. Между спинодалью и бинодалью заключена область метастабильных состояний, где сплав устойчив относительно малых флуктуаций состава, но претерпевает распад на равновесные фазы при появлении критических зародышей. Физической причиной существования этой области является вклад конфигурационной энтропии атомов в свободную энергию сплава.

В настоящее время считается установленным [3,4], что спинодаль является только теоретической концепцией. Она существует в пределе бесконечного дальнодействия [5-7], однако в системах с короткодействующим потенциалом тепловые флуктуации обеспечивают плавный переход от абсолютной неустойчивости к режиму зарождения и роста, так что невозможно определить линию, разделяющую эти два режима кинетики. Об этом свидетельствуют как экспериментальные данные $[8,9]$, так и результаты компьютерного моделирования $[10,11]$.

Процесс зародышеобразования вблизи бинодали менее изучен. В некоторых работах обсуждалась необходимость разделения области метастабильных состояний на две подобласти. Так, уже в [5] была построена схематическая фазовая диаграмма, включающая области „classical nucleation“ (сразу ниже бинодали) и „spinodal nucleation“ (сразу выше спинодали), хотя переход между этими областями предполагался плавным. В $[8,12]$ идея о двух режимах зарождения использовалась для объяснения экспериментов по рассеянию света в жидких бинарных смесях. В [13] было теоретически предска- зано, что между метастабильной областью и областью неустойчивых состояний существует третья область, где исходное однородное состояние устойчиво относительно бесконечно малых длинноволновых флуктуаций, но переходит к гетерофазному состоянию под действием конечных тепловых флуктуаций. Линия, ограничивающая сверху эту область, была названа физической спинодалью. В [14] подобная линия получена для однокомпонентного газа вблизи критической точки; было отмечено, что она лежит ближе к бинодали, чем к теоретической спинодали. В $[3,15]$ предсказано существование „псевдоспинодали“ в бинарных полимерных смесях, что нашло экспериментальное подтверждение [16] (отметим, однако, что речь идет об одиночных экспериментах). Псевдоспинодаль совпадает со спинодалью среднего поля (mean-field спинодалью) в пределе бесконечной молекулярной массы, что математически эквивалентно переходу к дальнодействующему потенциалу. Теория [3] справедлива при достаточно большой (хотя и конечной) молекулярной массе и не может быть перенесена на сплавы с короткодействующим потенциалом. Интерпретация псевдоспинодали дискуссионна. В [3] выдвигается гипотеза, что она разделяет области физически достижимых (наблюдаемых) и недостижимых (ненаблюдаемых) метастабильных состояний, потому что обычно распад реализуется быстро. Остается неясным, существует ли псевдоспинодаль в реальных сплавах с короткодействием; отсутствуют результаты моделирования методом Монте-Карло (МС), подтверждающие существование псевдоспинодали в каких-либо системах.

В настоящей работе на основе результатов МС-моделирования распада в сплаве с короткодействующим потенциалом показано, что гипотеза о существовании псевдоспинодали имеет под собой основания. Критерием достижения этой линии является устремление к бесконечности инкубационного периода возникновения критического зародыша, что, по-видимому, реализуется несколько ниже бинодали. Следовательно, псевдоспинодаль разделяет области, в первой из которых возможно 
гомогенное, а во второй - только гетерогенное зародышеобразование.

\section{2. Результаты моделирования}

Мы использовали простую схему кинетического МС, реализующую динамику Кавасаки [17] с прямым обменом атомами, ранее апробированную в $[18,19]$. Для моделирования был выбран эффективный парный потенциал $\mathrm{Cu}-\mathrm{Cu}$ в матрице ОЦК-Fе. Для простоты мы ограничились потенциалом в парамагнитном состоянии для первых трех координационных сфер $\{-7.4,-2.3,-0.3\} \mathrm{mRy}$ пренебрегая деформационной, концентрационной и магнитной поправками. Такой выбор не обеспечивает правильного предела растворимости $\mathrm{Cu}$ в ОЦК-Fе, но приводит к простой модели, достаточной для целей настоящей работы. Расчеты проводились для суперъячейки размером $90 \times 90 \times 90$ или $60 \times 60 \times 60$ элементарных ячеек с периодическими граничными условиями. Как и ранее в $[18,19]$, достигнутая к некоторому моменту времени степень распада $0<S<1$ рассчитывается из конфигурации атомов по правилу

$$
S=\frac{1}{N} \sum_{j=1}^{N} \Theta\left(\frac{1}{Z} \sum_{k=1}^{Z} \sigma_{k}^{(j)}-q\right),
$$

где $N$ - число атомов $\mathrm{Cu}$ в образце, $\sigma_{k}^{(j)}$ - числа заполнения для ближайших соседей вокруг узла $j$, $Z=8$ - координационное число для ближайших coceдей в ОЦК-решетке, $\Theta(x)$ - функция Хэвисайда. Согласно (1), атом $\mathrm{Cu}$ считается принадлежащим выделению, если локальная концентрация $\mathrm{Cu}$ в его окрестности не ниже $q$. Поскольку важно точно установить время старта распада, было принято $q=0.25$. В таком случае степень распада (1) чувствительна даже к появлению малых кластеров.

Как и ранее в [19], наши расчеты показывают отсутствие спинодали как физически выделенной линии, отделяющей область абсолютной неустойчивости от области зарождения и роста. С повышением температуры инкубационный период старта распада возрастает монотонно от весьма малых до очень больших значений (рис. 1). При этом мы обнаруживаем, что на технически достижимых временах расчета зарождение становится невозможным вблизи некоторой кривой на фазовой диаграмме, которая может быть названа псевдоспинодалью (либо пределом устойчивости гомогенного состояния) и которая заметно отличается от бинодали. Подобная кривая была ранее вычислена в [18] с использованием процедуры обычного Metropolis MC (с перестановкой атомов на произвольные расстояния) и ошибочно отождествлена с бинодалью, что привело авторов к некорректному выводу о согласии теории с экспериментом для предела растворимости $\mathrm{Cu}$ в ОЦК-Fе. Таким образом, требует тщательного анализа вопрос о том, возникает ли в наших расчетах предел устойчивости по

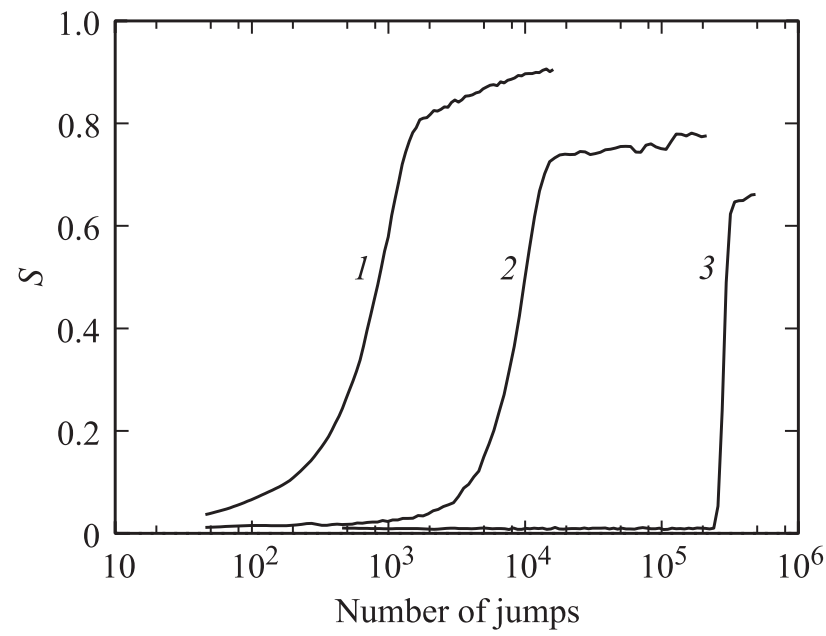

Рис. 1. Эволюция степени распада в сплаве с концентрацией примеси $c=0.015$ при $T=800$ (1), 1000 (2) и $1100 \mathrm{~K}$ (3). Время выражено числом скачков в расчете на один атом примеси.

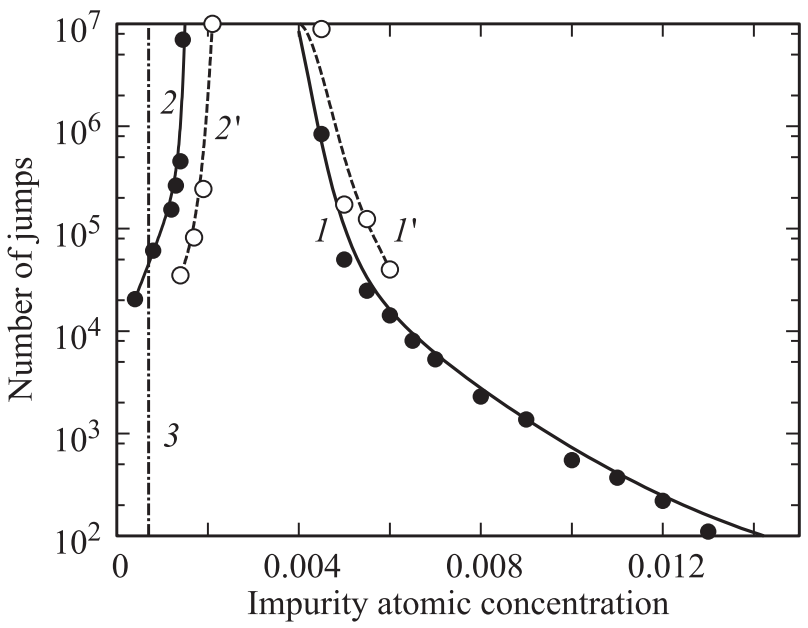

Рис. 2. Инкубационный период старта распада $\left(1,1^{\prime}\right)$ и время растворения выделения $\left(2,2^{\prime}\right)$ в зависимости от среднего состава сплава при температуре $T=800 \mathrm{~K}$. Размер расчетной области $90 \times 90 \times 90(1,2)$ или $60 \times 60 \times 60\left(1^{\prime}, 2^{\prime}\right)$ элементарных ячеек. Линия 3 соответствует равновесной концентрации примеси в матрице при контакте с крупным выделением. Время выражено числом элементарных перестановок в расчете на один атом примеси.

той причине, что большие времена расчета технически недостижимы, или он действительно является физически выделенной линией фазовой диаграммы.

Результаты наших расчетов при фиксированной температуре и вариации среднего состава представлены на рис. 2. Кривая 1 представляет инкубационный период старта распада в гомогенной системе, который определялся по условию достижения степени распада $S=0.05$. Кривая 2 представляет время растворения одиночного выделения при старте из двухфазного состояния, где все атомы примеси собраны в одно выделение в центре 


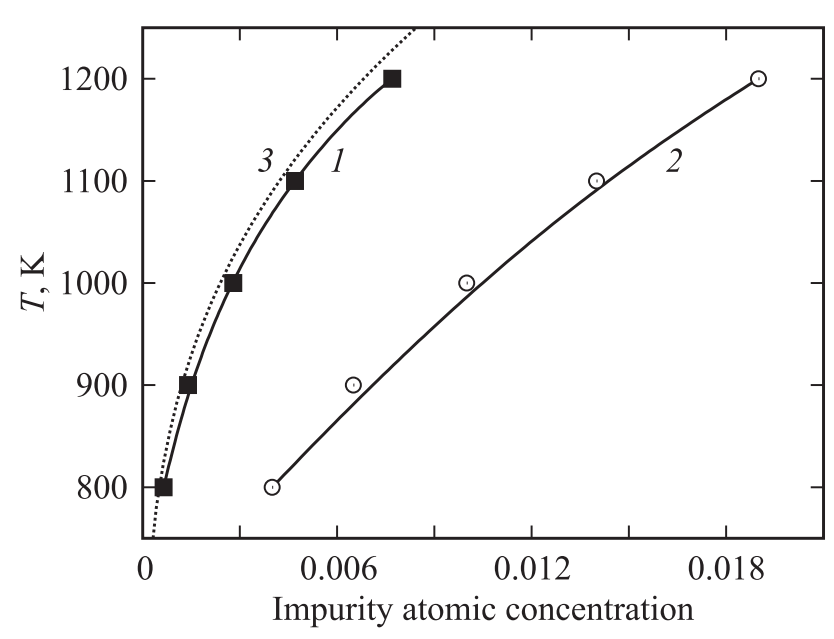

Рис. 3. Равновесная концентрация примеси в матрице (бинодаль) (1) и псевдоспинодаль, полученная из условия устремления инкубационного периода к бесконечности (2). Линия 3 построена с помощью формулы бинодали регулярного раствоpa [1].

расчетной области. Вертикальная линия 3 обозначает равновесную концентрацию примеси в матрице (бинодаль). Для расчета бинодали крупное выделение $\mathrm{Cu}$ помещалось в чистое железо, проводилась длительная выдержка до насыщения матрицы, затем рассчитывалась средняя концентрация примеси в слое матрицы, удаленном от исходного выделения.

Можно видеть, что время растворения выделения (кривая 2) устремляется к бесконечности вблизи равновесной концентрации в матрице (линия 3). Некоторое различие обусловлено эффектом Гиббса-Томсона $[20,21]$, поскольку кривая 2 строится для выделения небольшого размера, в то время как линия 3 соответствует равновесию массивных фаз. При этом инкубационный период старта распада (кривая 1), повидимому, устремляется к бесконечности при другой концентрации, которая выше равновесной в несколько раз. Учитывая, что для старта распада необходимо возникновение критического зародыша из тепловых флуктуаций, а максимально достижимый масштаб флуктуаций должен зависеть от размера расчетной области, мы построили аналогичные линии при уменьшении размера суперъячейки до $60 \times 60 \times 60$ (кривые $1^{\prime}, 2^{\prime}$ ). Можно видеть, что обе линии смещаются вправо примерно на одинаковую величину, поэтому различие пределов растворимости и устойчивости нельзя объяснить размерным эффектом.

На рис. 3 представлены температурные зависимости бинодали и псевдоспинодали на основании данных МС-моделирования (кривые 1 и 2 соответственно). Линия 3 соответствует бинодали регулярного твердого раствора [1], которая рассчитывается по формуле $k T=v(1-2 c) / \ln [c /(1-c)]$, где энергия смешения $v=-38.3 \mathrm{mRy}$ вычисляется из парного по- тенциала с учетом чисел заполнения координационных сфер, $v=\left(\sum_{i} z_{i} V_{i}\right) / 2, V_{i}=\{-7.4,-2.3,-0.3\} \mathrm{mRy}$, $z_{i}=\{8,6,12\}$. Можно видеть, что МС-бинодаль весьма близка к предсказанию модели регулярного раствора. Псевдоспинодаль в области малых концентраций близка к линейной функции, обнаруживая тем самым качественное сходство со спинодалью регулярного раствора, определяемой формулой $k T=-2 v c(1-c)$ [1]. Однако она имеет числовые значения на порядок ниже, чем спинодаль регулярного раствора, а по физическому смыслу (линия, на которой инкубационный период устремляется к бесконечности) заметно отличается от спинодали, обозначающей порог абсолютной неустойчивости сплава относительно малых длинноволновых флуктуаций. Заметим, что в работе [22] было показано, что спинодаль кластерного приближения (которое является более точным) лежит гораздо левее спинодали среднего поля, в то время как положение бинодали не изменяется.

\section{3. Обсуждение}

Предложим грубую качественную интерпретацию полученных результатов.

В отсутствие флуктуаций состава докритический зародыш $\left(R<R_{\mathrm{cr}}\right)$ растворяется, однако он может расти под действием флуктуации. Если при этом зародыш достигает критического размера $\left(R>R_{\mathrm{cr}}\right)$, он продолжает рост даже после прекращения действия флуктуации. С энергетической точки зрения вероятность возникновения флуктуации с энергией $\Delta G$ подчинена закону $\sim w_{0} \exp (-\Delta G / k T)$. Отсюда на первый взгляд следует появление критического зародыша при достаточной выдержке, если только $w_{0} \neq 0$. Однако формирование критического зародыша - процесс, развивающийся во времени, когда размер зародыша $R$ под действием флуктуации пробегает все мыслимые значения от одиночного атома до $R_{\mathrm{cr}}$. Поэтому наряду с энергетикой должны быть приняты во внимание кинетические аргументы. Максимальная скорость подвода нового вещества к зародышу в результате действия флуктуации ограничена средним составом сплава $c_{0}$ и уменьшается при понижении $c_{0}$. Напротив, интенсивность растворения докритического зародыша возрастает при приближении $c_{0}$ к бинодали. Два конкурирующих процесса уравновешиваются при некотором критическом размере зародыша.

Согласно [23], в отсутствие флуктуаций поток у поверхности зародыша

$$
j=\frac{D}{R}\left(c_{0}-c_{e}^{\infty}-\frac{\sigma}{R}\right),
$$

где $D-$ коэффициент диффузии, $\sigma-$ перенормированное поверхностное натяжение, $c_{e}^{\infty}-$ концентрация бинодали, а критический размер зародыша определяется условием $J=0$ :

$$
R_{\mathrm{cr}}=\sigma /\left(c_{0}-c_{e}^{\infty}\right)
$$


Введем в (2) дополнительный вклад - флуктуационный поток $D c_{0}$, действующий бесконечно долго, что соответствует образу максимально возможной флуктуации. Тогда условие для критического зародыша принимает вид

$$
R_{\mathrm{cr}}^{\text {fluc }}=\frac{\sigma}{\left(c_{0}-c_{e}^{\infty}\right)+c_{0} R_{\mathrm{cr}}^{\text {fluc }}} .
$$

В случае слабого пересыщения $\left(c_{0}-c_{e}^{\infty} \gg 1\right)$ имеем

$$
R_{\mathrm{cr}}^{\mathrm{fluc}}=\sqrt{\sigma / c_{0}}
$$

Величина $R_{\mathrm{cr}}^{\text {fluc }}$ остается большой при достаточно малом $c_{0}$, поэтому в слабо пересыщенных сплавах вблизи бинодали должна существовать область, где докритические зародыши растворяются даже в условиях действия максимально возможного флуктуационного потока. Значит, для появления критического зародыша в этой области необходима дополнительная стимуляция, т.е. реализуется только гетерогенное зарождение.

\section{4. Заключение}

Таким образом, на основании результатов МС-моделирования мы высказываем гипотезу о существовании на фазовой диаграмме бинарного сплава псевдоспинодали (или предела устойчивости однородного состояния относительно тепловых флуктуаций состава). Эта линия разделяет области гомогенного и гетерогенного зародышеобразования.

Автор признателен Ю.Н. Горностыреву и И.Г. Шмакову за полезные обсуждения.

\section{Список литературы}

[1] Дж. Кристиан. Теория превращений в металлах и сплавах. Мир, М. (1978). 806 с.

[2] А.Г. Хачатурян. Теория фазовых превращений и структура твердых растворов. Наука, М. (1974). 384 с.

[3] Z.-G. Wang. J. Chem. Phys. 117, 481 (2002).

[4] G. Wilemski, J.-S. Li. J. Chem. Phys. 121, 7821 (2004).

[5] K. Binder. Phys. Rev. A 29, 341 (1984).

[6] W. Klein, C. Unger. Phys. Rev. B 28, 445 (1983).

[7] C. Unger, W. Klein. Phys. Rev. B 29, 2698 (1984).

[8] B. Chu, F.J. Schoenes, M.E. Fisher. Phys. Rev. 185, 219 (1969).

[9] R.J. Speedy, C.A. Angell. J. Chem. Phys. 65, 851 (1976).

[10] K. Binder. Ann. Phys. 98, 390 (1976).

[11] D.W. Heermann, W. Klein, D. Stauffer. Phys. Rev. Lett. 49, 1262 (1982).

[12] G.B. Benedek. In: Polarisation matie et rayonnement, Livre de Jubile en l'Honneur du Professor A. Kastler. Universitaires de Paris, Paris (1968). P. 71.

[13] А.З. Паташинский, Б.И. Шумило. ФТТ 22, 1126 (1980).

[14] S.B. Kiselev, I.G. Kostyukova. J. Chem. Phys. 98, 6455 (1993).

[15] S.M. Wood, Z.G. Wang. J. Chem. Phys. 116, 2289 (2002).

[16] A.A. Lefebvre, J.H. Lee, N.P. Balsara, B. Hammouda. J. Chem. Phys. 116, 4777 (2002).
[17] K. Kawasaki. In: Phase transitions and critical phenomena / Eds C. Domb, M.S. Green. Academic, N.Y. (1972). V. 2. P. 443.

[18] O.I. Gorbatov, I.K. Razumov, Yu.N. Gornostyrev, V.I. Razumovskiy, P.A. Korzhavyi, A.V. Ruban. Phys. Rev. B 88, 174113 (2013).

[19] И.Г. Шмаков, И.К. Разумов, О.И. Горбатов, Ю.Н. Горностырев, П.А. Каржавый. Письма в ЖЭТФ 103, 119 (2016).

[20] Р.А. Свелин. Термодинамика твердого состояния. Металлургия, М. (1968). 316 с.

[21] D. Perez, L. Lewis. Phys. Rev. E 74, 031609 (2006).

[22] K.Yu. Khromov, F. Soisson, A.Yu. Stroev, V.G. Vaks. ЖЭТФ 139, 479 (2011).

[23] Л.Д. Ландау, Е.М. Лифшиц. Теоретическая физика. Т. Х. Физическая кинетика. Наука, М. (1979). 528 с. 